\title{
Evolución y evaluación tecnológica de los protocolos de comunicaciones
}

\author{
L.R. Vega-González, J. Avilés-Zúñiga, M. Montalvo-Taboada \\ Centro de Ciencias Aplicadas y Desarrollo Tecnológico, CIADET, \\ y Facultad de Ingeniería, UNAM. \\ E-mails:lrvg@servidor.unam.mxymtmau@latinmail.com
}

(recibido: octubre de 2002; aceptado: enero de 2003)

\begin{abstract}
Resumen
En los años sesenta del siglo pasado, con el advenimiento y uso del transistor en las computadoras, se inició la llamada revolución de la información. Su principal característica ha sido la intensa tasa de cambio en la innovación, la cual ha permitido avances sin precedentes y la convergencia de los sistemas de información dedicados con los sistemas de telecomunicaciones. Este fenómeno se intensificó aún más durante las dos últimas décadas del siglo pasado, cuando la tecnología de Internet abrió la puerta a un nuevo orden mundial, dando paso a un mundo globalizado en el que la transmisión de voz, datos y video se lograron con toda facilidad entre dos puntos cualquiera del globo terráqueo. Tal vez las tecnologías que mayormente han contribuido a estos grandes impactos económicos y sociales han sido los protocolos de comunicaciones. En este trabajo se realiza una revisión de la evolución de los mismos y se propone su evaluación tecnológica relativa con base a sus dimensiones tecnológicas. Finalmente, se discute la propuesta prospectiva de un protocolo digital estándar que seguramente permitirá un sistema de redes en todas partes, generando la interconexión absoluta del mundo en el futuro cercano.
\end{abstract}

Descriptores: protocolos de comunicación, tecnología, evolución y evaluación.

\begin{abstract}
The information revolution started with the transistor invention, and its integration and use in the computers during the sixth decade of the last century. Immediately unprecedented advances followed the intense innovation rate of change bringing telecommunication dedicated information systems convergence. This phenomenon was intensified during the very last decades of last century, when Internet technologies opened the door to a new world order bringing a global world in which voice, data and video transmission could be easily done between any two points of the earth. May be the technologies that mainly contributed to these great economical and social impacts have been the communication protocols. In this work we review its evolution and propose a method for its technological evaluation based in its technological dimensions. Finally the prospective proposal of a standard digital protocol is discussed. It will surely permit a "Network Everywhere" System leading absolute world interconnectivity in the near future.
\end{abstract}

Keywords: communication protocols, technology evolution, evaluation. 


\section{Convergencia de los sistemas de información dedicados con los sistemas de telecomunicaciones}

Con la existencia de procesadores y memorias de ultra alta escala de integración y software de aplicación complejo, a mediados de los ochenta del siglo pasado, proliferó la aparición de sistemas dedicados con muy diversas aplicaciones en los ámbitos de procesos industriales, de saludy manufactura, entre muchos otros.

Un caso particular en esta evolución lo constituyen los sistemas de instrumentación y control en los que la filosofía pasó del control de lazo sencillo a los grandes sistemas de control distribuido, capaces de manejar cientos o miles de lazos para sistemas tan complejos como los procesos de toda una refinería. Este concepto de grandes sistemas de control no tuvo éxito debido a las grandes dificultades y costos implicados en el mantenimiento de la confiabilidad y la disponibilidad de los sistemas. Con el paso del tiempo, la tendencia logró ser de micro sistemas de control, dedicados a la supervisión de ciertas partes específicas de los procesos distribuidos a lo largo y ancho de las plantas. Con esto sobrevino un gran problema, los sistemas de control eran muchas veces de diferentes fabricantes, quienes se habían preocupado por el desarrollo de protocolos de comunicación propietarios, pero que no podían comunicarse con otros, ni por supuesto, con otros sistemas o instrumentos de marcas diferentes, por lo que los subprocesos permanecían aislados.

De esta manera, aparecieron una infinidad de protocolos y medios de transmisión (buses) como el DeviceNet, el Profibus, el micromax de L\&N, el TDC de Honeywell, el Fox de Foxboro, etcétera. Prácticamente todos los fabricantes contaban con sus propios protocolos para la inteconexión de sus sistemas. A estos esquemas de trabajo se les llamó "sistemas cerrados".

Ante esta situación, la cual generaba problemas y costos adicionales, fue necesario que las asociaciones de instrumentistas internacionales trabajaran en un estándar para lograr la completa interconexión de los sistemas hacia una filosofía de sistemas abiertos.
Nacieron así los estándares "fieldbus" para la interconexión física y el protocolo de comunicación HART. Hoy por hoy existen instrumentos individuales de campo inteligentes como las válvulas inteligentes de Masoneilan (3) o los transmisores inteligentes de Rosemount (4) entre muchos otros, con la capacidad de poder ser monitoreados y calibrados a distancia a través de este protocolo.

Actualmente los controladores Lógicos Programables o PLC's se han desarrollado alrededor de los conceptos de sistemas abiertos, software abierto, comunicaciones abiertas y son capaces de manejar los protocolos TCP/IP logrando comunicaciones a través de Internet, con lo que permite la interconexión de sistemas geográficamente distantes en configuraciones de redes WAN (Cleaveland, 2000). De esta forma, es posible la supervisión de procesos, instrumentos individuales o sistemas entre el headquarters de una fábrica y sus aplicaciones en cualquier lugar remoto del mundo.

Otro ejemplo de este tipo de sistemas de información dedicados, lo constituye la instrumentación biomédica de uso en laboratorios de investigación y hospitales, éste tuvo un desarrollo muy-similar, ya que decenas de fabricantes como Beckmnan, Kodak, Scientific Instruments y Varian, entre muchos otros, desarrollaron equipos con capacidades de intercomunicación a centros de cómputo locales y posteriormente a PC's. La idea de proveer servicios médicos a distancia, también motivó la evolución hacia los sistemas abiertos. Actualmente se habla de hospitales totalmente digitales como el Georges Pompidou en Paris, en el cual todas las operaciones diarias son registradas y mantenidas por computadora a través de un sistema de registro electrónico de pacientes (EPR) que hace que todos los reportes médicos, resultados de laboratorio e imágenes, estén disponibles instantáneamente para los médicos, en donde quiera que ellos se encuentren. El sistema corre en un backbone de fibra óptica en modo de transferencia asíncrona (ATM) de $622 \mathrm{Mb} / \mathrm{s}$ que alimenta la red de área local LAN del hospital. 
También hay un enlace por medio de un enrutador a una red privada de hospitales, la cual está conectada a Internet, (Weiss, 2002). Para finales del siglo pasado, la evolución de los "sistemas cerrados" a "sistemas abiertos" permitió la convergencia de los sistemas de información dedicados con los sistemas de telecomunicaciones.

\section{Evolución tecnológica de los protocolos de comunicaciones}

Las telecomunicaciones han dado paso a la interconectividad de los sistemas abiertos. La base de esta interconectividad la constituyen los protocolos. (Spanier, 1998). Para el desarrollo de este trabajo se han revisado distintos aspectos relativos a los protocolos de comunicaciones que van desde aspectos relacionados con la historia, su origen, aplicación e incluso algunos actores que han intervenido en el desarrollo y difusión de algunas tecnologías.

Se consideraron aspectos técnicos específicos para cada tipo de protocolo tomando como marco de referencia varios aspectos. En primer lugar, la forma de cómo trabaja el protocolo a partir del modelo OSI de siete capas y el modelo de red consistente en tres grandes secciones: los equipos terminales que accesan o inician una sesión de comunicaciones, la nube o red de transporte y los equipos terminales que reciben la comunicación. También se tomaron en cuenta los "medios físicos de transmisión" con lo que se complementaron los aspectos requeridos para la comprensión de la forma en que trabajan los protocolos dentro de las redes de comunicaciones.

Tabla 1. Resumen de la evolución de los protocolos de comunicación para redes de telecomunicaciones y sus características

\begin{tabular}{|c|c|c|c|c|c|c|c|c|}
\hline $\begin{array}{c}\text { Protocolo } \rightarrow \\
\text { Características } \downarrow\end{array}$ & NCP & HDLC & IP & TCP & X.25 & $\begin{array}{l}\text { FRAME } \\
\text { RELAY }\end{array}$ & ATM & IPV6 \\
\hline Desarrollado por & ARPANET & OSI & ARPANET & ARPANET & $\begin{array}{l}\text { Compañías } \\
\text { Telefónicas }\end{array}$ & $\begin{array}{l}\text { Evolución de } \\
\text { X.25 }\end{array}$ & $\begin{array}{l}\text { AT\&I/France } \\
\text { Telecomm }\end{array}$ & IETF \\
\hline Fecha & 1968 & 1970 & 1973 & 1973 & 1975 & 1980 & 1985 & En desarrollo \\
\hline $\begin{array}{l}\text { Tecnología de } \\
\text { conmutación }\end{array}$ & Paquetes & Paquetes & Paquetes & Paquetes & Paquetes & Paquetes & $\begin{array}{l}\text { Paquetes y } \\
\text { emulación de } \\
\text { circuitos }\end{array}$ & $\begin{array}{c}\text { Conmutación } \\
\text { de circuitos } \\
\text { WDM }\end{array}$ \\
\hline $\begin{array}{l}\text { Equipos } \\
\text { terminales }\end{array}$ & $\begin{array}{l}\text { Teléfonos y } \\
\text { Mainframes }\end{array}$ & $\begin{array}{c}\text { PC'sy } \\
\text { Teléfonos }\end{array}$ & $\begin{array}{l}\text { PC'sy } \\
\text { Teléfonos }\end{array}$ & $\begin{array}{l}\text { PC'sy } \\
\text { Teléfonos }\end{array}$ & $\begin{array}{l}\text { PC'sy } \\
\text { Teléfonos }\end{array}$ & $\begin{array}{l}\text { PC's } \\
\text { Teléfonos y } \\
\text { PBX }\end{array}$ & $\begin{array}{c}\text { Compuvideo y } \\
\text { Teléfonos }\end{array}$ & $\begin{array}{c}\text { Compuvideo y } \\
\text { Teléfonos }\end{array}$ \\
\hline Conmutación & $\begin{array}{l}\text { Centrales } \\
\text { analógicas }\end{array}$ & $\begin{array}{l}\text { Centrales } \\
\text { analógicas }\end{array}$ & $\begin{array}{l}\text { Centrales } \\
\text { digitales } \\
\text { (inicio) }\end{array}$ & $\begin{array}{l}\text { Centrales } \\
\text { digitales } \\
\text { (inicio) }\end{array}$ & $\begin{array}{l}\text { Centrales } \\
\text { dgitales y } \\
\text { analógicas }\end{array}$ & $\begin{array}{l}\text { Centrales } \\
\text { digitales }\end{array}$ & $\begin{array}{l}\text { Centrales } \\
\text { digitales }\end{array}$ & $\begin{array}{l}\text { Centrales } \\
\text { digitales }\end{array}$ \\
\hline Acceso & Vía MODEM & $\begin{array}{l}\text { Vía } \\
\text { MODEM }\end{array}$ & $\begin{array}{l}\text { Digital con } \\
\text { NTU's }\end{array}$ & $\begin{array}{l}\text { Digital con } \\
\text { NTU's }\end{array}$ & $\begin{array}{l}\text { Vía } \\
\text { MODEM y } \\
\text { digital }\end{array}$ & Digital & Digital & Digital \\
\hline Objetivo & $\begin{array}{l}\text { Transferen- } \\
\text { cia de } \\
\text { archivos entre } \\
\text { computado- } \\
\text { ras }\end{array}$ & $\begin{array}{c}\text { Encapsula } \\
\text { miento y } \\
\text { transferen- } \\
\text { cia de } \\
\text { datos } \\
\text { utilizando } \\
\text { lineas } \\
\text { telefónicas } \\
\text { analógicas }\end{array}$ & $\begin{array}{l}\text { Transmitir } \\
\text { información } \\
\text { en forma de } \\
\text { paquetes sin } \\
\text { control de } \\
\text { flujo }\end{array}$ & $\begin{array}{l}\text { Entrega y } \\
\text { recepcion de } \\
\text { información } \\
\text { de mannera } \\
\text { confiable }\end{array}$ & $\begin{array}{l}\text { Encapsula- } \\
\text { miento y } \\
\text { transporte de } \\
\text { datos usando } \\
\text { líneas telef. } \\
\text { analógicas }\end{array}$ & $\begin{array}{l}\text { Encapsula- } \\
\text { miento y } \\
\text { transporte de } \\
\text { voz y datos }\end{array}$ & $\begin{array}{l}\text { Encapsulamiento } \\
\text { y transporte de } \\
\text { voż, datos e } \\
\text { imágenes en } \\
\text { cualquier } \\
\text { protocolo }\end{array}$ & $\begin{array}{l}\text { Comunicación } \\
\text { Global de } \\
\text { cualquier tipo } \\
\text { de red }\end{array}$ \\
\hline Tipo de tráfico & Daṭos & Datos & Datos & Datos & Datos & Voz y Datos & $\begin{array}{l}\text { Todo tipo de } \\
\text { tráfico }\end{array}$ & $\begin{array}{l}\text { Todo tipo de } \\
\text { tráfico }\end{array}$ \\
\hline $\begin{array}{c}\text { Capa de } \\
\text { interacción en el } \\
\text { modelo OSI }\end{array}$ & No aplica & 2 y 3 & 3 & 4 & 2 y 3 & 2 & 2 & 2 \\
\hline Tipo de Servicio & $\begin{array}{l}\text { Orientado a } \\
\text { NO conexión }\end{array}$ & $\begin{array}{l}\text { Orientado } \\
\text { a NO } \\
\text { conexión }\end{array}$ & $\begin{array}{l}\text { Orientado a } \\
\text { NO } \\
\text { conexión }\end{array}$ & $\begin{array}{l}\text { Orientado a } \\
\text { conexión }\end{array}$ & $\begin{array}{l}\text { Orientado a } \\
\text { conexión }\end{array}$ & $\begin{array}{l}\text { Orientado a } \\
\text { conexión }\end{array}$ & $\begin{array}{l}\text { Orientado a } \\
\text { conexión }\end{array}$ & $\begin{array}{l}\text { Orientado a } \\
\text { conexión }\end{array}$ \\
\hline Aplicación & $\begin{array}{c}\text { Redes } \\
\text { militares e } \\
\text { investigación }\end{array}$ & $\begin{array}{l}\text { Interco- } \\
\text { nexión de } \\
\text { redes } \\
\text { geográfica- } \\
\text { mente } \\
\text { distantes } \\
\text { (WAN) de } \\
\text { bajas vel. } \\
\end{array}$ & Internet & Internet & $\begin{array}{l}\text { Interco- } \\
\text { nexión de } \\
\text { redes LAN }\end{array}$ & Redes WAN & $\begin{array}{c}\text { Redes de alta } \\
\text { velocidad en Voz, } \\
\text { datos y Video }\end{array}$ & $\begin{array}{l}\text { Transporte de } \\
\text { cualquier tipo } \\
\text { de tráfico } \\
\text { usando } \\
\text { tecnologías } \\
\text { ópticas }\end{array}$ \\
\hline
\end{tabular}


El resultado del estudio de los protocolos de comunicaciones más importante de las últimas décadas se presenta en la tabla 1 que muestra un resumen detallado de sus características tomando como referencia la línea de tiempo.

\section{Variables impulsoras económicas y sociales}

Indudablemente el origen del desarrollo explosivo de los protocolos de comunicaciones tuvo su origen como muchas otras tecnologías en la segunda guerra mundial, ya que la investigación desarrollada en las universidades para los gobiernos Norteamericano, Inglés y de otros países europeos, respondía a la necesidad de evitar la pérdida de comunicaciones en caso de eventos de guerra. Así, fue desarrollado Arpanet, el predecesor del protocolo Internet. La intensa difusión y uso para correo electrónico y el intercambio global de datos se presentó como una gran oportunidad de mercado para las empresas de servicios "Carriers" que pasaron a ser los siguientes actores que impulsaron el desarrollo de nuevos protocolos, ya que invirtieron en mucho equipamiento para establecer grandes "Backbonds" que pudieran servir como la red de transporte de las cada vez más demandadas comunicaciones, lo cual, por supuesto, redundaría en aumento de sus ganancias por cuestiones tarifarias.

Los impulsores sociales han sido el interés creciente de los distintos sectores sociales para formar parte de un nuevo sistema mundial en el que la mayoría de los individuos quieren usar un teléfono celular, sistemas de localización personal, sistemas de navegación geográfica e inclusive a través de interfases humanas buscar la convergencia de la tecnología en el hogar de acuerdo con Battelle (1).

\section{Variables tecnológicas impulsoras}

Hadi y Holland (2000), consideran que desde 1997 las variables impulsoras tecnológicas en el desarrollo de productos de alta tecnología, como son los robots, han sido el creciente poder de los microprocesadores, la reducción de costos de procesamiento digital en paralelo, la disponibilidad de ambientes operativos para PC multitarea, la disponibilidad y la madurez del software de procesamiento gráfico.

Todo esto aplica a los protocolos de comunicaciones con algunas cuestiones adicionales.

En primer lugar, de acuerdo con el resumen de la tabla 1, el desarrollo de los protocolos puede remontarse hasta fines de los años sesenta del siglo pasado, por lo que su desarrollo se vio influenciado por el avance y aumento en las distintas escalas de integración de los procesadores y las memorias dinámicas. Por supuesto, la madurez relativa del software modular disponible ha sido contribuyente al desarrollo.

Por su parte, la disponibilidad de cada vez mejores interfases físicas y su normalización, ha brindado un apoyo fundamental para la confiabilidad, disponibilidad y calidad de los servicios. Actualmente se encuentran disponibles tecnologías de medios de transmisión muy confiables como la fibra óptica de alta velocidad y nuevos dispositivos como los interruptores ópticos y opto electrónicos que han eliminado los cuellos de botella en las transmisiones, a través de anchos de banda cada vez más grandes que pronto llegarán al orden de gigabytes. Por medio de estos dispositivos será posible la transmisión de todo tipo de tráfico con excelente calidad y definición (Dailey, 2001).

En la actualidad, la tendencia es hacia el desarrollo de dispositivos ópticos de nueva tecnología, como las unidades "cross connect" que serán la base de las redes con backbone óptico requeridas en las tecnologías "Wave División Multiplex" (WDM) que muy probablemente sean las líderes del futuro (Metz, 2000), Cisco, Service Provider (2), (Muñiz, 2001).

\section{Evaluación tecnológica}

Toda evaluación tecnológica tiende a ser subjetiva, ya que depende de los objetivos de la misma. Medellín y Vega (1995), propusieron como elementos básicos para una evaluación tecnológica la calificación de una serie de "aspectos críticos" entre los cuales se encuentran 
algunos relacionados con el grado de integración del paquete tecnológico, el mercado o aspectos financieros de las empresas que desarrollan la tecnología. Posteriormente, propusimos la inclusión de los efectos del ciclo de vida de la tecnología en su evaluación (Vega, 2001).

En el caso de los protocolos de comunicaciones, se trata de tecnologías ya desarrolladas, probadas $y$ difundidas plenamente, por lo que son tecnologías que están, o bien, en la parte alta del ciclo de vida, es decir, en la fase de "estabilización" o en la etapa de declinación o decrecimiento.

Por otra parte, la evaluación requiere hacerse de forma relativa entre las distintas tecnologías, por lo que, para eliminar la gran complejidad y la subjetividad en la metodología de evaluación, se requirió de la definición precisa de las variables tecnológicas que conforman las dimensiones tecnológicas de las tecnologías bajo estudio. Enseguida presentamos el método desarrollado para realizar la evaluación.

\section{Metodología de evaluación}

La tabla 1 resume las características relevantes de la evolución de los protocolos de comunicaciones a partir de la década de los sesenta del siglo pasado. Para realizar la cuantificación de la posición relativa de los diferentes protocolos de comunicaciones en nuestro país, hemos considerado que una buena aproximación de las dimensiones tecnológicas de todo protocolo de comunicaciones debe tomar en cuenta las variables tecnológicas que fueron clave en el desarrollo y difusión de las tecnologíás. Es decir, en la metodología de evaluación se utilizaron las variables impulsoras discutidas en los primeros puntos.

Una medida indirecta del impacto de las variables impulsoras tecnológicas en el desarrollo de los protocolos de comunicaciones, lo es $\sin$ duda, el tipo de tráfico manejado (calidad de servicio) y el tipo o plataforma de tecnología integrada. Por otra parte, según Lucky (2002), no existe un solo fabricante o proveedor de servicios (carrier) que sea quien maneje el reloj maestro de sincronía del Internet, por lo que no podemos obtener un buen indicador de los impactos sociales y económicos en México, a través de medir el crecimiento de las empresas de telecomunicaciones ubicadas en nuestro país. Por lo anterior, se considera que un buen indicador, también indirecto, de alguna forma ha provocado impactos sociales y económicos en el desarrollo de los protocolos, valoración subjetiva del uso y difusión que ha tenido cada tipo de protocolo en nuestra región geográfica específica. Por esta razón, fue considerada la variable "aplicación en México" como la tercera variable comprendida dentro de las dimensiones tecnológicas que también nos permitió realizar una cuantificación relativa.

Es válido utilizar la variable "aplicación en México" con propósitos de cuantificación, debido a la subjetividad de este tipo de análisis, ya que esta variable es una medida de la difusión relativa que ha tenido determinado tipo de protocolo en nuestro medio.

La figura 1 presenta el resumen de las dimensiones tecnológicas analizadas, las cuales son de tipo de tráfico, tipo de tecnología y aplicación en México, éstas a su vez tienen que ver con las variables tecnológicas impulsoras económicas (E), tecnológicas ( $T$ ) y sociales $(S)$, discutidas anteriormente.

A partir de la tabla 1 se obtienen las ponderaciones de cada protocolo. La evaluación tecnológica realizada es relativa en términos de una calificación máxima de 10.0 asignada al protocolo ideal de comunicaciones; es decir, aquel que cuente con las mayores atribuciones posibles.

\begin{tabular}{|c|c|c|c|c|c|}
\hline \multicolumn{5}{|c|}{ Dimensiones Tecnológicas } \\
\hline $\begin{array}{c}\text { Tipo de } \\
\text { Tráfico }\end{array}$ & \multicolumn{2}{|c|}{$\begin{array}{c}\text { Tipo de } \\
\text { Tecnología }\end{array}$} & \multicolumn{2}{|c|}{$\begin{array}{c}\text { Aplicación en } \\
\text { México }\end{array}$} \\
\hline S & T & T & E & E & S \\
\hline
\end{tabular}

figura 1. Variables usadas para la Evaluación Tecnológica 
El Protocolo Digital Ideal (PDI) deberá tener una calificación máxima de $33.33 \%$ para la variable "Tipo de Tráfico"; es decir, deberá ser capaz de manejar datos, voz, video, así como todo tipo o clase de información que pueda surgir en el futuro. El PDI deberá también tener una calificación máxima de $33.33 \%$ para la variable "Tipo de tecnología" ya que deberá contar con los últimos avances en capacidad de procesamiento, software multitasking paralelo y medios de transmisión que permitan un ancho de banda prácticamente infinito. Finalmente, la calificación asignada para el PDI deberá ser de 33.33\% para la variable "Aplicación en México", ya que deberá ser el protocolo de mayor uso y difusión, y probablemente encapsulará y sustituirá todos los protocolos previos.

Los valores usados para las variables integrantes de las dimensiones tecnológicas de los protocolos de comunicación, considerando el PDI como referencia máxima, se presentan en la tabla 2. A partir de estos valores se ponderará cada protocolo.

\section{HDLC}

Este protocolo fue creado para transmitir datos a través de centrales telefónicas de conmutación analógicas, con las siguientes características:

Tipo de Tráfico: Datos.

Tipo de Tecnología: Conmutación de paquetes a través de centrales telefónicas analógicas.

Aplicación en México: Casi nula, ya que resulta ser un protocolo de datos obsoleto.

\section{$\underline{X .25}$}

Es un protocolo para comunicaciones a nivel WAN, que define cómo se establecen y mantienen las conexiones entre los dispositivos de usuario y de red.

Tipo de Tráfico: Datos.

Tipo de Tecnología: Conmutación de paquetes a través de centrales telefónicas analógicas. Aplicación en México: Comercial, bancaria y gubernamental.

Frame Relay

Protocolo WAN que opera en las capas 1 y 2 del modelo OSI, se considera una evolución de X.25 Tipo de tráfico: Voz, datos y video.

Tipo de Tecnología: Conmutación de paquetes.

Aplicación en México: Bancarias, comerciales y gubernamentales entre otras.

\section{$\mathrm{TCP} / \mathrm{IP}$}

TCP corresponde a la capa 4 de OSI, su función es el control de flujo y la transmisión confiable de la información.

IP corresponde a la capa 3 de OSI, su función es el ruteo de paquetes a través de la red.

Tipo de Tráfico: Datos.

Tipo de Tecnología: Conmutación de paquetes en entornos LAN y WAN.

Aplicación en México: académica, comercial, gubernamental y bancaria, entre otras.

\section{ATM}

Es una tecnología de conmutación de celdas que reúne los beneficios de la conmutación de circuitos y la conmutación de paquetes.

Tipo de Tecnología: conmutación de paquetes y emulación de circuitos sólo en sistemas digitales.

Tabla 2. Desglose de variables de evaluación y peso respectivo

\begin{tabular}{|c|c|c|c|c|c|}
\hline \multicolumn{2}{|c|}{ Tipo de táfico } & \multicolumn{2}{|c|}{ Tipo de Tecnología } & \multicolumn{2}{|c|}{ Aplicación en México } \\
\hline Voz & $8.3325 \%$ & Analógica & $11.11 \%$ & Académica & $6.67 \%$ \\
\hline Datos & $8.3325 \%$ & $\begin{array}{l}\text { Conmutación de } \\
\text { paquetes }\end{array}$ & $11.11 \%$ & Bancaria & $6.67 \%$ \\
\hline Video & $8.3325 \%$ & $\begin{array}{l}\text { Conmutación de } \\
\text { circuitos }\end{array}$ & $11.11 \%$ & Industrial & $6.67 \%$ \\
\hline \multirow[t]{2}{*}{ Imágenes } & $8.3325 \%$ & & & Comercial & $6.67 \%$ \\
\hline & & & & Gubernamental & $6.67 \%$ \\
\hline Totales & $33.33 \%$ & & $33.33 \%$ & & $33.3 \%$ \\
\hline
\end{tabular}


Tipo de Tráfico: todo tipo de tráfico. Aplicación en México: académica y bancaria.

\section{IPV6}

Protocolo de capa 3 que a diferencia del IPV4 manejará 16 octetos para el direccionamiento, además cuenta con calidad de servicio y autentificación.

Tipo de Tecnología: conmutación de paquetes.

Tipo de Tráfico: todo tipo de tráfico.

Aplicación en México: académica.

La tabla 3 presenta los resultados numéricos de la evaluación, incluyendo la calificación relativa de los diferentes protocolos estudiados respecto al Protocolo Digital Ideal.

\section{Mapa tecnológico}

El proceso de evaluación tecnológica desarrollado en la sección anterior, culmina con la calificación de los protocolos, relativa al mejor protocolo posible, es decir, al Protocolo Digital Ideal. Los resultados se muestran en la última columna de la tabla 3.

Graficando las calificaciones relativas para cada protocolo que se encuentran en la tercera columna de la tabla 3 , contra el tiempo aproximado en el que se considera la aparición en el mercado del mismo, obtenemos un mapa tecnológico que nos muestra la posición relativa, generada a partir de su evaluación tecnológica entre los protocolos.

El mapa tecnológico de la figura 2, permite identificar con una flecha de línea continua aquellos protocolos que encapsulan a otros; con una flecha de línea discontinua los protocolos que evolucionaron directamente a partir de otros y con flecha con línea de punto y guión los protocolos que en la actualidad tienden a tomar el liderazgo tecnológico hacia el protocolo digital estándar que probablemente aparecerá en el mercado entre la segunda y la tercer década de este siglo.

\section{E1 Protocolo Digital Estándar (SDP) y la Convergencia Absoluta}

El desarrollo y la evolución tecnológica de los protocolos de comunicaciones mostrada en el mapa tecnológico de la figura 2 fue derivada de las variables impulsoras tratadas en los puntos de variables impulsoras, económicas y sociales y variables tecnológicas impulsoras; esto permite concluir que la convergencia de computadoras y comunicaciones (C\&C) presentada por Saito (1997) hacia el año 2000 , se cumplió perfectamente, pero ha sido rebasada en su concepción y tenderá a convertirse en una Convergencia Absoluta, en la cual no sólo se unirán los sistemas de cómputo y comunicaciones,

Tabla 3. Ponderación y calificación relativa de los protocolos de comunicaciones

\begin{tabular}{|c|c|c|c|c|c|}
\hline Protocolo & $\begin{array}{c}\text { Tipo de Tráfico } \\
0-33.33 \% \\
\end{array}$ & $\begin{array}{c}\text { Tipo de Tecnología } \\
0-33.33 \%\end{array}$ & $\begin{array}{c}\text { Aplicación en México } \\
0-33.33 \%\end{array}$ & \multicolumn{2}{|c|}{$\begin{array}{l}\text { Calificación ponderada } \\
\text { del Protocolo }\end{array}$} \\
\hline HDLC & Datos $=8.3325 \%$ & $\begin{array}{l}\text { Conmutación paquetes } \\
\text { en WAN }=11.11 \%\end{array}$ & $\begin{array}{l}\text { Casi nula (obsoleto) }= \\
6.67 \%\end{array}$ & $26.10 \%$ & $\rightarrow 3.00$ \\
\hline X.25 & Datos $=8.3325 \%$ & Igual anterior $=11.11 \%$ & $\begin{array}{l}\text { Comercial + Bancaria }+ \\
\text { Gubernamental }= \\
6.67 * 3=20.01\end{array}$ & $39.45 \%$ & $\rightarrow 4.0$ \\
\hline Frame Relay & Datos $=8.3325 \%$ & $\begin{array}{l}\text { Conmutación de } \\
\text { paquetes }=11.11 \%\end{array}$ & $\begin{array}{l}\text { Igual al anterior + otras } \\
=33.33 \%\end{array}$ & $69.44 \%$ & $\rightarrow 7.0$ \\
\hline TCP/IP & $\begin{array}{l}\text { Voz }+ \text { datos }+ \text { Video }= \\
8.3325 * 3=25 \%\end{array}$ & $\begin{array}{l}\text { Conmutación paquetes } \\
\text { en entornos LAN } \\
\text { WAN }=22.22 \%\end{array}$ & $\begin{array}{l}\text { Igual al anterior }+ \\
\text { Académica }=33.33 \%\end{array}$ & $63.88 \%$ & $\rightarrow 6.4$ \\
\hline ATM & Datos $=8.3325 \%$ & $\begin{array}{l}\text { Conmutación de } \\
\text { paquetes y circuitos } \\
22.22 \%\end{array}$ & $\begin{array}{l}\text { Académica y Bancaria = } \\
6.67^{*} 2=13.34 \%\end{array}$ & $68.89 \%$ & $\rightarrow 7.0$ \\
\hline IPV6 & $\begin{array}{l}\text { Todo tipo de Tráfico = } \\
33.33 \%\end{array}$ & $\begin{array}{l}\text { Conmutación de } \\
\text { paquetes y circuitos } \\
22.22 \%\end{array}$ & $\begin{array}{l}\text { Sólo Académica = } \\
6.67 \%\end{array}$ & $62.22 \%$ & $\rightarrow 6.2$ \\
\hline Protocolo Digital Ideal & $33.33 \%$ & $33.33 \%$ & $33.33 \%$ & $100 \%$ & $\rightarrow 10.0$ \\
\hline
\end{tabular}


sino todas las tecnologías de la información. La investigación prospectiva desarrollada por diversos grupos como el dirigido por Halal (1993) de la Universidad de George Washington, quien estima que para el año 2020 estará listo un protocolo de comunicaciones que será conocido como Protocolo Digital Estándar (Standard Digital Protocol, SDP).

Por otra parte, el artículo "The Network in Eevery Room", publicado el mes de Febrero del 2002, Wayt (2001) señala que ahora ya es posible trabajar con redes en el hogar, cuyo medio de transmisión son las líneas de potencia eléctrica. Esto significa que estamos ante sistemas tecnológicos extraordinarios que han logrado vencer el eterno problema del "ruido" en los datos e información, el cual puede presentarse por la presencia de voltajes y corrientes de potencia. Esto ha sido posible a través de software muy especializado con códigos de prevención de error que corren sobre procesadores de muy alta integración. La complejidad en el procesamiento de estas señales excede considerablemente todo lo utilizado hasta el día de hoy y abre la puerta a un mundo interconectado totalmente.

Si los medios de transmisión y el ruido no son ya un problema, entonces estas tecnologías actuarán también como impulsoras del desarrollo del Protocolo Digital Estándar. En la figura 3 se presenta la prospección de algunas trayectorias tecnológicas que probablemente nos llevarán a la convergencia absoluta por medio del PDE.

\section{El futuro: redes en todas partes (Nets Everywhere)}

La convergencia absoluta significa que la tecnología de telecomunicaciones llegará e inundará nuestros hogares. Recientemente Levy (2001), en la sección "Focus on Technology" de Newsweek propuso una visión de cómo serán los hogares del futuro, resalta una pantalla plana gigante de ultra alta definición que podrá conmutar entre video, Internet o fotos familiares, así como un control remoto universal para prácticamente utilizar todas las cosas de la casa, aparatos de línea blanca como refrigerador o la lavadora conectados a red y que proporcionarán información sobre las corridas de lavado o la existencia de ciertos alimentos. Sistemas de audio conectados inalámbricamente a instrumentos musicales.

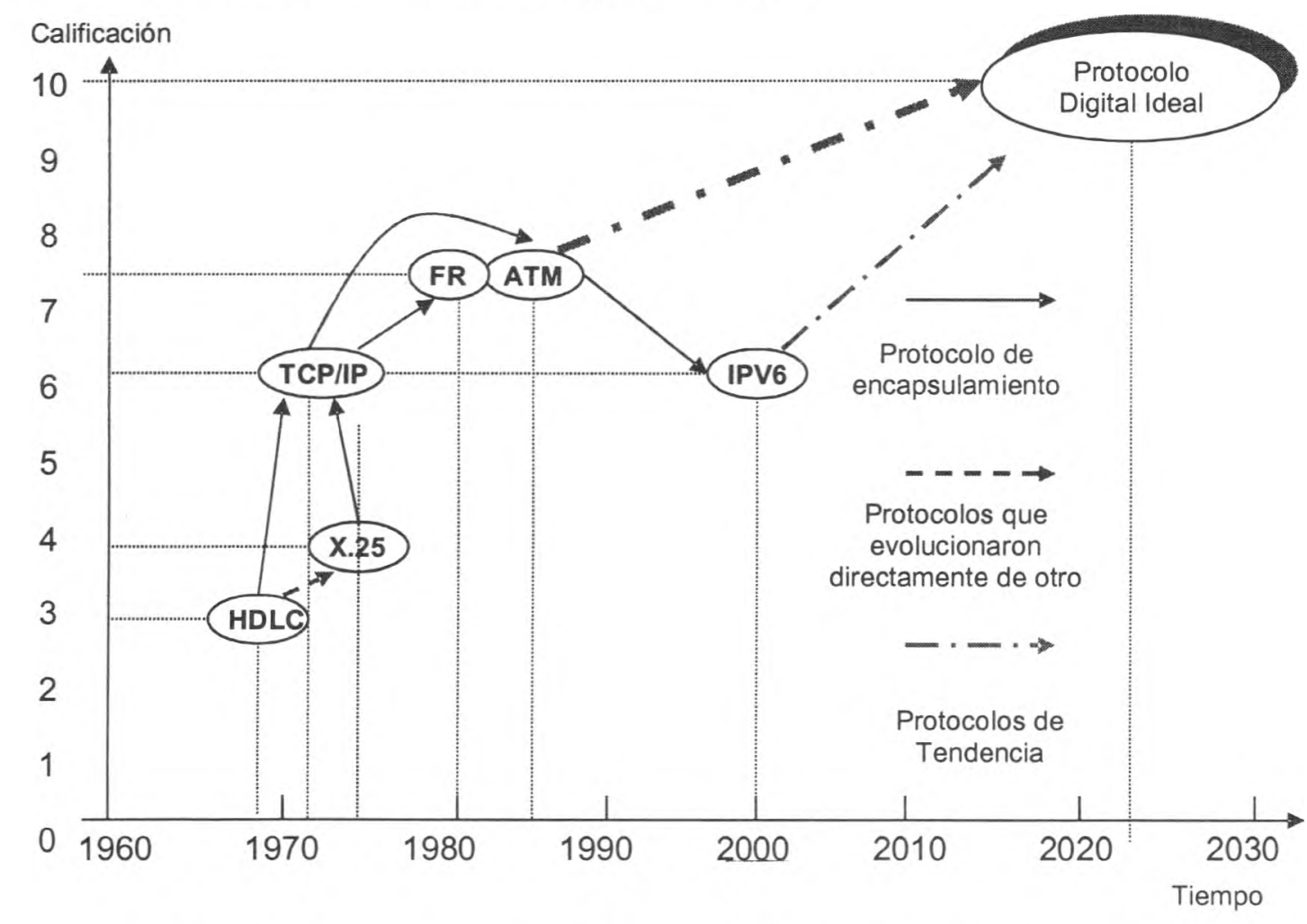

Figura 2. Mapa tecnológico de la evolución de los protocolos de comunicación 


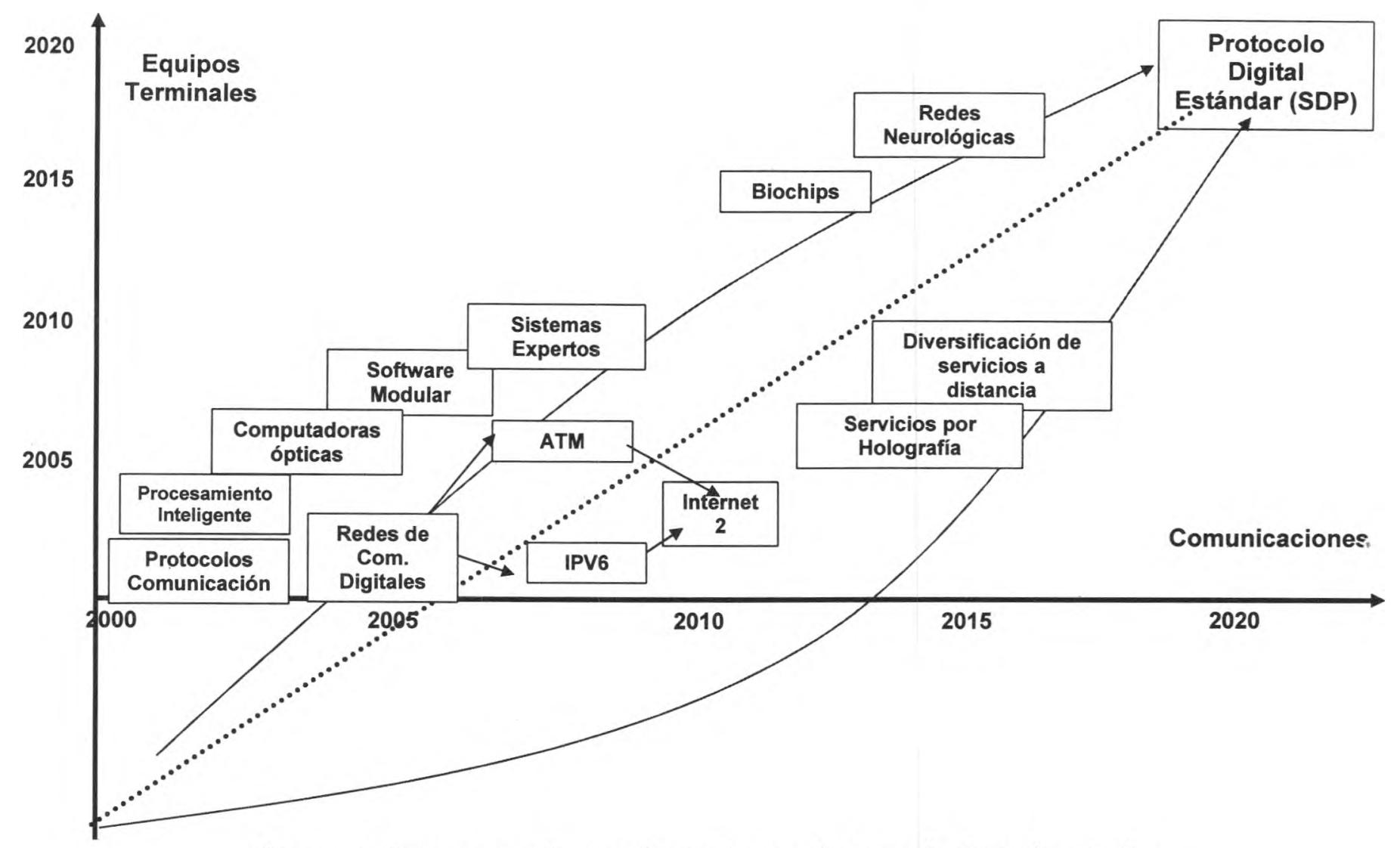

Figura 3. Prospectiva de tecnologias hacia el protocolo digital estándar

Fuente: desarrollado por información de estudios prospectivos realizados en la Universidad George Washington. Halal (Op. Cit.)

Por otra parte, la sociedad asimilará más el comercio electrónico (E Business \& Electronic Shopping). El trabajo en casa (Work at home) será una realidad a través del correo electrónico avanzado, teléfonos móviles, mini LabTops, pantallas de video conferencia. Por otra parte, la educación tenderá cada vez más a hacerse a distancia. Muchos de estos dispositivos y sistemas ya existen en la actualidad, pero aún no están enlazados todos en una misma red, y la calidad de servicio (CS) que se recibe en muchos de los casos, aún es muy deficiente. Sin embargo, es de esperarse que en el corto plazo, el ancho de banda de los sistemas de transmisión y la puesta a punto de las tecnologías de los protocolos digitales ópticos, mejoren extraordinariamente la CS proporcionando comunicaciones optimas a velocidades no sospechadas.

Lo anterior nos hace pensar que probablemente tienen razón quienes piensan que esto nos llevará al "nuevo orden mundial" propuesto por Halal et al. (1998) en el que una de las principales características será el aislamiento cada vez mayor de los seres humanos; sin embargo, los futurólogos no se alarman, ya que se estima que para hablar con otro ser humano que se encuentre a muchos kilómetros de distancia sólo será necesario iniciar una sesión de holovisión, la cual será instantánea debido a las extraordinarias capacidades de los sistemas de comunicación que transmitirán la información de video a través del protocolo digital estándar a la velocidad del orden de gigabites por segundo, usando como medio de transporte la luz.

Lo deseable sería que la sociedad y la humanidad en su conjunto se encuentren preparadas para asimilar los intensos cambios que vienen en las siguientes décadas. Se podría ser optimista en el sentido de que esta revolución tecnológica marcará el inicio de una nueva etapa, la cual beneficiará a más seres humanos, esperando con ello, defender la vida social y evitando la soledad que nos quita la posibilidad de compartir el calor y las emociones de otros seres humanos en nuestra proximidad. 


\section{Conclusiones}

1. Uno de los resultados de este trabajo es el mapa tecnológico que nos indica la posición relativa de los diferentes protocolos de comunicaciones más usados y que han determinado el estado del arte en el área. En particular, el mapa permite comparar el desempeño tecnológico de los más importantes protocolos de comunicación utilizados en México.

2. Nuestros resultados indican, que la convergencia de los sistemas de computadoras y comunicaciones que predijo Saito (1997), ha sido rebasada en su alcance, ya que no sólo han convergido estos dos tipos de sistemas, sino que en un par de décadas, prácticamente todo sistema que sea parte de la vida humana se convertirá en parte de una gran red mundial.

3. Los estudios prospectivos consultados, señalan que la base de la gran red mundial será el protocolo digital estándar. Este permitirá la interconexión entre todo tipo de sistemas con la mejor calidad de servicio. La evolución de los sistemas de comunicaciones estará marcada por las tecnologías en las que se basarán los equipos terminales.

4. Las dimensiones tecnológicas usadas para la evaluación tecnológica fueron determinadas cuantitativamente a partir de la definición de las variables impulsoras tecnológicas, sociales y económicas en el contexto mexicano. Este método permitió un posicionamiento relativo de las diferentes tecnologías que han sido utilizadas en nuestro país, dentro del gran contexto mundial.

5. El estudio señala que dentro de los protocolos más demandados y difundidos hoy en día se encuentran por supuesto la familia TCP-IP, Frame Relay y existe una fuerte tendencia a migrar hacia ATM. Sin embargo, las tecnologías ópticas de transporte están ganado terreno, por lo que se espera que IPV6 e Internet II se difundirán en breve, en los tiempos que permitan los costos de acceso.

6. Los gradientes que determinan la dinámica del desarrollo tecnológico crecen vertiginosamente. Los impactos individuales y sociales no se han hecho esperar, al grado de que en menos de dos décadas la mayoría de los estilos de vida han cambiado profundamente.
7. Una tarea pendiente es la de identificar hasta qué punto el ser humano, sus organizaciones y sociedades podrán adaptarse a los cambios por venir y plantear las acciones de adaptación pertinentes.

\section{Referencias}

Batelle Institute (1) www. batelle.gov

Cisco Service Provider: Optical Internetworking (2) http://www.cisco.com/warp/public/779/serv pro/solutions/optical/docs/whatiswdm

Cleavelan P. (2000). Programmable Controllers Stay Current, Special Report, I\& CS, May, pp23-42.

Dailey P.L. (2001). Optical Switches Turn to Holography. Computer, Vol. 24, No. 11, pp 20.

Hadi A.A. y Holland S.W. (2000). Product and Technology Trends for Industrial Robots, Industrial Robots Simposium. Proceedings of the 2000 IEEE International Conference on Robotics \& Automation, San Francisco, California, EUA, April.

Halal W.E. (1993). The Information Technology Revolution: Computer Hardware, Software and Services for the $21^{\text {st }}$ Century. Technology Forecasting \& Social Change, Vol. 44, pp 69-86, Elsevier Science Inc.

Halal W.E., Kull M.D., Leffman A. (1998). The George Washington University Forecast of Emerging Technologies: A Continuous Assesment of the Technology Revolution. Technology Forecasting \& Social Change, Vol. 59, pp 89-110. Elsevier Science Inc.

Levy S. (2001). The Next New Thing. Newsweek, December 17, pp 61-64.

Lucky R. W. (2002). Who Runs the Internet? IEEE SPECTRUM, March, pp80.

Medellín C.E., Vega G.L.R. (1995). Evaluación de tecnología, herramienta de gestión útil para la transferencia. Anais do XV Congresso Brasileiro de Engenharia de Produção, Universidade Federal de São Carlos Brasil. Vol. 2, pp 731-735.

Masoneilan (3): ValVue, valve communication software Data Sheet.www.masoneilan.com

Metz C. (2000). IP Over Optical, From Packets to Photons, IE EE Internet Computing, http://computer.org/internet/ 
Munis Bandeira O.C. (2001). IP over WDM, Mestrado em Teleinformática e Automação, Seminario Redes de Computadores COE728, Universidades Federal de Río de Janeiro, Brasil, http://www.gta.ufrj.br/fagundes/IP WDM/ip wdm.htm

Rosemount (4): Smart Transmitter 3344P, Profibus http://www.rosemount.com/document/pds/4021b00n.pdf

Saito F. (1997). Managing Technology development at NEC Corporation. International Journal of Technology Management, Vol. 14, No 2/3/4, pp196-207.

Spanier S. (1998). Tecnologías de interconectividad de redes. Prentice Hall, México.

Vega G.L.R. (2001). Evaluación, avalúo y ciclo de vida de la tecnología, Parte I, presentada para su revisión y arbitraje a la revista Ingeniería, Investigación y Tecnología, Mayo del 2001.

Wayt G.B. (2001). The network in every room.

Scientific American, Vo. 286, No.2, pp 28-33

Weiss G. (2002). Welcome to the (almost) digital Hospital, IEEE SPECTRUM, Mach, pp44-48.

\section{Bibliografía sugerida}

Deasington R. J. (1990). X.25 Explained. Ellis Horwood.

Faynbero I. (1997). The Intelligent Network Standards. Mc. Graw Hill.

Schwartz M. (1998). Telecommunication Networks, Protocol, Modeling \& Analysis. Addison Wesley

Smith P. (1993). Frame Relay Principles and Applications. Addison Wesley.

\section{Semblanza de los autores}

Luis Roberto Vega-González. Es ingeniero mecánico electricista por la Facultad de Ingeniería de la UNAM. Obtuvo la maestría en ingeniería de sistemas en el área de planeación por la DEPFI-UNAM, y la maestría en administración de las organizaciones por la División de Estudios de Posgrado de la Facultad de Contaduría y Administración de la UNAM. Asimismo, se especializó en gestión de la innovación tecnológica por el Centro para la Innovación Tecnológica y la FCyA de la UNAM. Durante más de veinte años colaboró con diversas firmas nacionales e internacionales en proyectos de las áreas de instrumentación, control y potencia. Actualmente es coordinador de vinculación y gestión tecnológica del Centro de Ciencias Aplicadas y Desarrollo Tecnológico (CCADET) de la UNAM.

Javier Avilés-Zúñiga. Se gradúa como ingeniero eléctrico electrónico por la Facultad de Ingeniería de la UNAM con la tesis: Estudio Evolutivo, Evaluación Tecnológica y Prospectiva de los Protocolos de Comunicaciones y su Impacto en el Mundo Actual, en julio de 2002. Es profesor en el Colegio de Ciencias y Humanidades, Plantel Sur en el área de ciencias experimentales. Actualmente es asesor de primero y segundo semestre de la materia de física, en el Colegio de Ciencias y Humanidades.

Mauricio Montalvo-Taboada. Se graduó como ingeniero eléctrico, electrónico en el año 2002 por la Facultad de Ingeniería de la UNAM. Actualmente se desarrolla en el diseño y manejo de redes de telecomunicaciones e integración de redes WAN y LAN en corporaciones internacionales. Ha laborado en monitoreo de redes corporativas de Nestlé, Hérdez, AMEX, etc., así como en monitoreo de enlaces de microondas de alta capacidad de servicios remotos, aislamiento y diagnóstico remoto de problemas de interconexión, utilizando el software de redes 46020 Network Managment y HP Openview con equipos Newbridge. 